\title{
Multi-image Super Resolution Reconstruction with Pepper and Salt Noise
}

\author{
Fengqing Qin ${ }^{1}$, Lilan Cao, Lihong Zhu, Wanan Yang
}

\begin{abstract}
To improve the resolution and reduce the noise in image, a framework of Multi-image super resolution reconstruction with Pepper and Salt noise is proposed. In the low resolution imaging model, the processes of movement, blur, down-sampling and Pepper and Salt noise are considered. Firstly, for the multiple low resolution images, the Pepper and Salt noise in each image is reduced through median filtering method. Then, super resolution reconstruction is carried out on the multiple de-noised low resolution images through iterative back projection algorithm. Experiments show that the visual effect and the peak signal to noise ratio (PSNR) of the super resolution reconstructed image is improved greatly.
\end{abstract}

Keywords: Multi-image; Super resolution; Pepper and salt noise; Median filter; Iterative back projection.

\section{Introduction}

Super resolution (SR) refers to reconstructing a high resolution (HR) image from one or multiple low resolution (LR) images, which is an efficient way to increase the spatial resolution with lower cost than hardware method.

According to the number of the LR images, image SR mainly includes multiimage SR and single-image SR [1-3]. As there are more complementary information can be used, multi-image SR [4][5] is commonly researched to produce a HR image based on a set of images that were acquired from the same scene [6]. The degrading processes mainly include movement between the scene and the camera, the blur of the imaging system, the down sampling and system noise. In many papers, the movement with sub-pixel precision is estimated and utilized to reconstruct a HR image [7] [8]. The blurring process is considered in some paper to improve the quality of the SR image [9].

\footnotetext{
${ }^{1}$ Fengqing Qin $(\bowtie)$

Institute of Computer Science and Technology in Yibin University, Yibin, China, 644000

e-mail: qinfenqing@163.com
} 
In most of the SR reconstruction algorithms, the process of noise is seldom considered, which restrained the quality of the SR reconstructed image. The noise can worsen the quality of images and bring some difficulty to image analysis. Thus, noise should be considered in the framework of single-image SR reconstruction.

In this paper, a framework of multi-image SR reconstruction method with Pepper and Salt noise is proposed. In the LR imaging model, the processes of movement, blur, down-sampling and noise are all considered. The Pepper and Salt noise is reduced through median filtering method, and SR reconstruction is performed on the de-noised low resolution image by iterative back projection (IBP) algorithm.

\section{Framework of multi-image SR reconstruction with Pepper and Salt noise}

The framework of multi-image SR reconstruction with Pepper and Salt noise is shown in Fig.1. Firstly, the original LR image is denoised through median filter. Then, SR image is reconstructed through iterative back projections algorithm.

\subsection{The LR Imaging Model}

According to the imaging process, the movement, blur, down-sample and noise are considered in the LR imaging model, as shown in Fig.1. The mathematical description of LR imaging model of multi-image SR reconstruction may be expressed as follows:

$$
Y=E B D F+N
$$

Where, $F$ represent the HR image; $Y$ represents the LR images; $E$ is the movement process; $B$ is the blur function; $D$ is the down-sample process; $N$ is the noise.

Firstly, the HR image $(F)$ is transformed by different movement parameters. Here, vertical and horizontal shift of camera are considered. Secondly, the transformed images are blurred by convolving with a point spread function (PSF). Gaussian blur is the most common and is considered here. Thirdly, the blurred images are down-sampled by a given integer factor. Here, the down-sampled images are gained by taking the neighborhood average gray value of the blurred images. Finally, the down-sampled images are noised, and the final LR images are gained. Here, the Pepper and Salt noise is considered. 


\subsection{Iterative back projection method}

Among the current SR reconstruction methods, the iterative back projection (IBP) method has the virtues of small computational amount, fast convergent rate, good reconstruction effect, and so on. In addition, the estimated information about the LR imaging model can be well utilized in the IBP algorithm. If the LR imaging model is estimated more accurately, the SR reconstructed image will achieve better quality.

In IBP algorithm, by back projecting the estimation error between the estimated LR image and the original image onto the HR image grid, the estimation error is gained to modify to estimated HR image. Repeating the above process until the iteration time is greater than a given number or the estimation error is less than a threshold, the SR image will be gained.

According to this idea, the IBP algorithm may be expressed as follows:

$$
\hat{f}_{k+1}=f_{k}-\lambda H^{B P}\left(\hat{y}_{k}-y\right)
$$

Here, the initial value of the estimated HR image $\left(\hat{f}_{0}\right)$ is taken as the interpolated image of the reference LR image by Bilinear interpolation algorithm.

\subsection{Median filter algorithm}

The median filter algorithm is widely used due to good smoothing performance for noise with long-tailed probability distribution and some image detail preserving capability. It is used to remove Pepper and Salt noise here.

In median filter algorithm, the isolated noise points are eliminated by making the gay values be close to their real values. The gray value at a given point is replaced by the median gray value in the sliding window. In the sliding window, the gray values are sorted in ascending or descending way. The median filter may be expressed as follows:

$$
y(i, j)=\operatorname{med}\left\{y_{0}(i-m, j-n),(m, n \in W)\right\}
$$

Where, $y_{0}(i, j)$ is the noised image; $y(i, j)$ is the denoised image; $W$ is a two dimensional sliding window. The sliding window is often taken as a $K \times K$ rectangle, where $K$ is often an odd number.

\subsection{Framework of Multi-image SR}

According to the LR imaging model proposed in this paper and the idea of IBP algorithm, the framework of the multi-image SR reconstruction method with noise is shown in Fig.1. Here, $\mathrm{k}$ is the iteration time; $\hat{f}$ is the estimated SR image; $y$ is 
the observed LR image; $\hat{y}$ is the simulated LR images of $\hat{f}$ passed through the LR imaging model; $B$ and $D$ are the matrix forms of the motion blur and downsampling respectively; $n$ is the system noise; $E^{-1}, B^{-1}, D^{-1}$ and $n^{-1}$ denote the inverse operation of $E, B, D$ and $n ; H^{B P}$ is the back projection operation; $\hat{y}-y$ is the difference of simulated LR image and the denoised LR image; $\lambda$ is the gradient step.

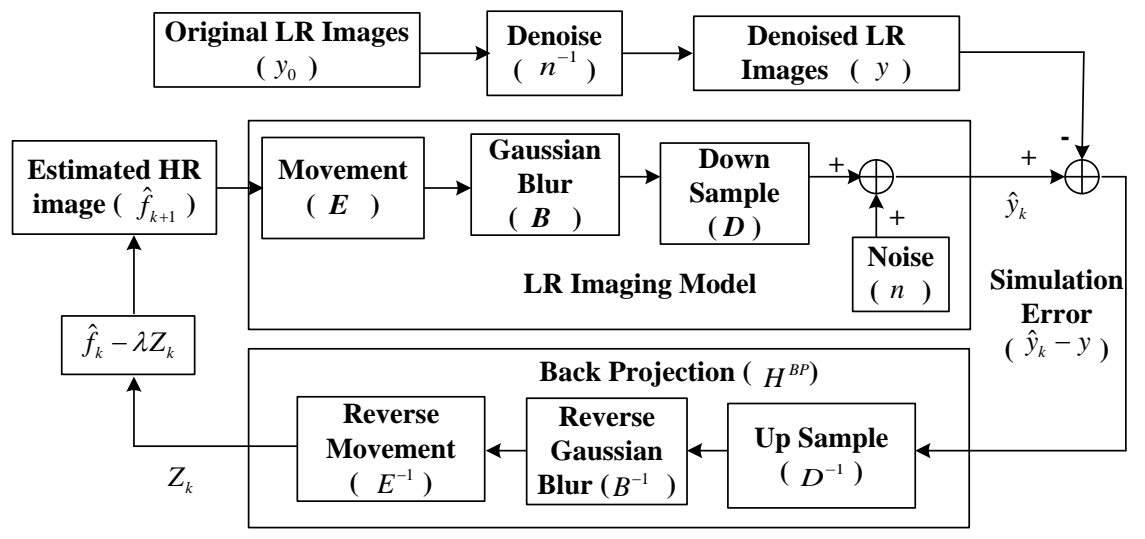

Fig. 1 Framework of Multi-image SR reconstruction with noise

\section{Experiments}

Experiments are performed on simulated LR image to test the algorithm objectively and subjectively. In order to avoid boundary effects caused by movement, the test image 'lena.bmp' of size $256 \times 256$ pixels as shown in Fig. 1 is added with a zero-window zero window with size of 16 pixels around it, which results in a simulated high-resolution image of size $288 \times 288$ pixels as shown in Fig. 3 .

The simulated HR image in Fig. 3 is passed through the LR imaging model as shown in Fig.1 to simulate 5 LR images. Firstly, the HR image is shifted horizontally and vertically with a range of $[-5,5]$ pixels respectively. The movement parameters are shown in table 1 . Secondly, the 5 transformed images are convolved by a Gaussian PSF with size of 7 and standard deviation of 0.1 respectively. Thirdly, the blurred images are down-sampled by a factor of 2 in horizontal and vertical direction. Finally, Pepper and Salt noise with density of 0.1 is added. The generated LR images with size $144 \times 144$ are shown in Fig.4. 


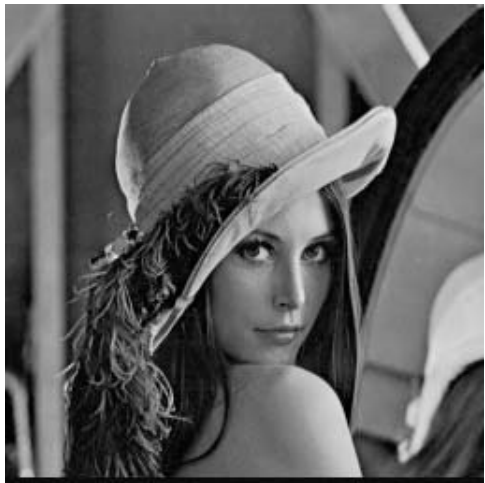

Fig.2 Original HR image.

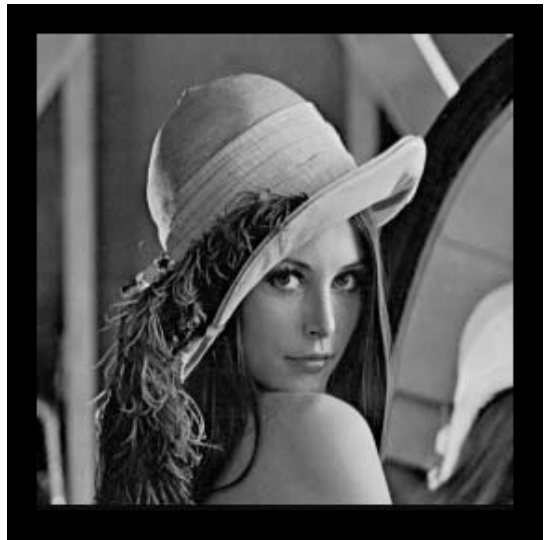

Fig.3 Simulated HR image.

Table 1 The movement parameters in horizontal and vertical directions.

\begin{tabular}{|c|c|c|c|c|c|}
\hline Parameters & $\boldsymbol{E}_{\mathbf{1}}$ & $\boldsymbol{E}_{\mathbf{2}}$ & $\boldsymbol{E}_{\mathbf{3}}$ & $\boldsymbol{E}_{\mathbf{4}}$ & $\boldsymbol{E}_{\mathbf{5}}$ \\
\hline Horizontal shift & 0 & -4.3487 & 2.4547 & -2.6452 & 4.3456 \\
\hline Vertical shift & 0 & -2.5463 & -3.5671 & 3.2747 & -3.6857 \\
\hline
\end{tabular}

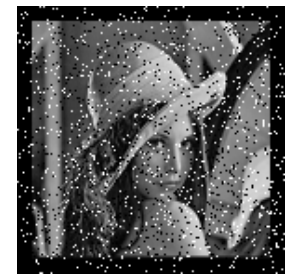

(a)

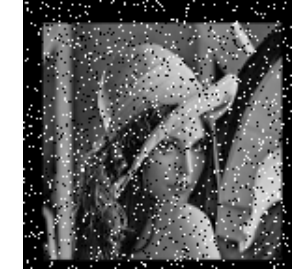

(b)

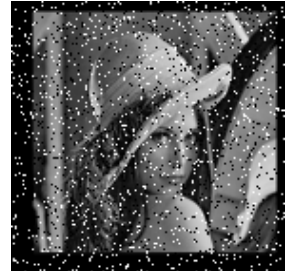

(c)

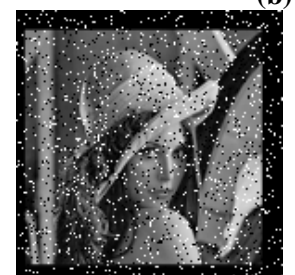

(d)

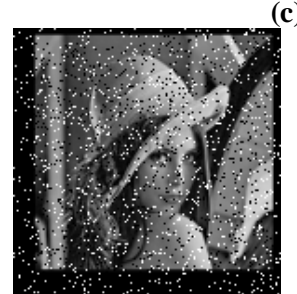

(e)

Fig.4 The simulated LR images.

Taking the first LR image as the reference image, using the method proposed in this paper, the SR reconstructed image is shown in Fig.5. The peak signal to noise ratio (PSNR) of the SR image is $34.5077 \mathrm{~dB}$. If image denoising process isn't performed, the SR of the LR image, and the 2 times Bilinear interpolated image of the denoised reference LR image is shown in Fig.6. The 2 times Bilinear interpolated image of the LR image is shown in Fig.7. The mean square error (MSE) and PSNR of these three methods are shown in Table 2. 
The experimental results show the effectiveness of the proposed method. The Pepper and Salt noise is restrained in the SR reconstructed image. The SR reconstructed image has better visual effect and higher PSNR than other two methods.

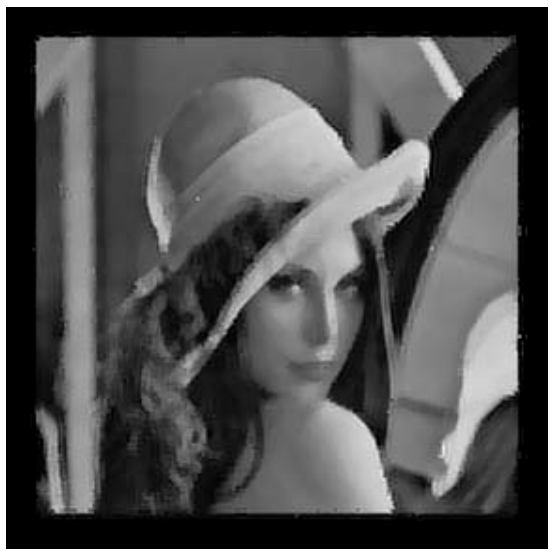

Fig.5 The SR reconstructed image of the denoised LR images.

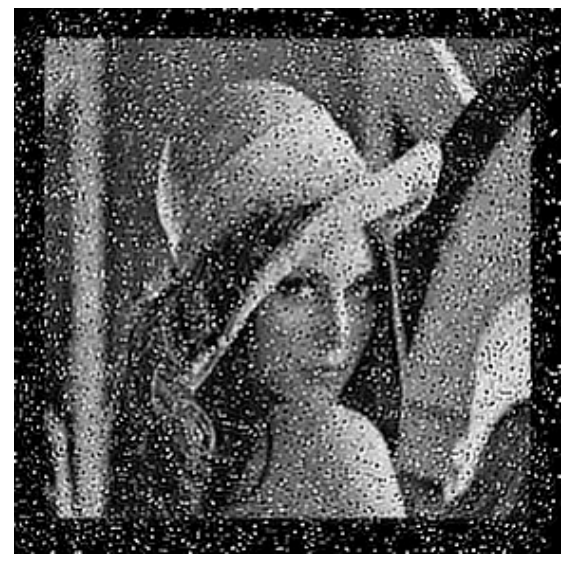

Fig.7 The SR reconstructed image of the LR images.

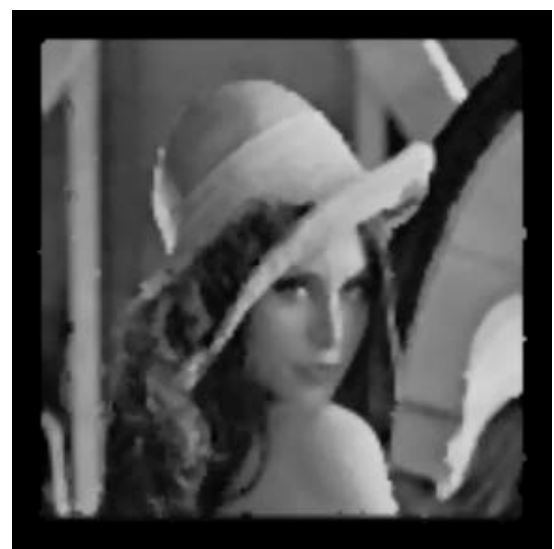

Fig.6 The interpolated image of denoised reference LR image.

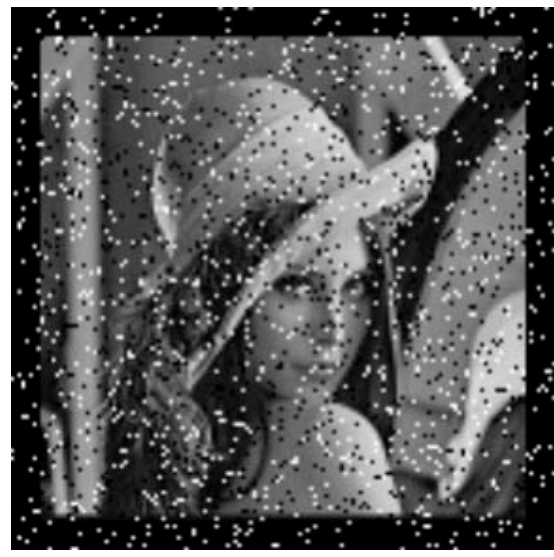

Fig.8 The interpolated image of the reference LR image.

Table 2. The MSE and PSNR of different methods.

\begin{tabular}{|c|c|c|c|c|}
\hline $\begin{array}{c}\text { Performance } \\
\text { parameters }\end{array}$ & Fig.5 & Fig. 6 & Fig.7 & Fig.8 \\
\hline MSE & 23.0307 & 24.7700 & 69.6092 & 34.7155 \\
\hline PSNR (in dB) & 34.5077 & 34.1915 & 29.7041 & 32.7256 \\
\hline
\end{tabular}




\section{Summary}

A framework of multi-image SR reconstruction with Pepper and Salt noise is proposed. The degrading processes of movement, blur, down sample and noise are considered in the LR imaging model. The gained LR image is denoised by median filter. The SR image is reconstructed through IBP algorithm, in which the LR imaging processes are considered. The Pepper and Salt noise are well restrained in the SR reconstructed image, and the PSNR is improved. In future work, how to reserve the detail information when removing noise will be researched to make further efforts in improving the SR reconstructed image.

\section{Acknowledgements}

This paper is supported by the National Nature Science Foundation of China (Gant no.61202195, 61202196), the Sichuan Provincial Education Department project (Gant no.11ZA174, 11ZA223, 11ZA224), the Application Fundamental Research Project of Sichuan Provincial Scientific and Technology Department (Gant no.2011JY0139), the key project of Yibin Science and Technology Bureau (Gant no.2011SF016).

\section{References}

1. Kim K I, Kwon Y (2010) Single-image super-resolution using sparse regression and natural image prior. IEEE Transactions on Pattern Analysis and Machine Intelligence, 32(6)11271133

2. Yang S Y, Wang M, Chen Y G, Sun Y X (2012) Single-image super resolution reconstruction via learned geometric dictionaries and clustered sparse coding. IEEE Transactions on Image Processing, 21(9): 4016-4028

3. Chris D (2011) Single image super-resolution using self-examples and texture synthesis. SIViP, 5:343-352

4. Zhang L, Yuan Q Q, Shen H F, Li P X (2011) Multifrmae image super-resolution adapted with local spatial information, J. Opt. Soc. Am A, 28(3):381-390

5. Giannoula A (2011) Classification-Based Adaptive Filtering for Multiframe Blind Image Restoration. IEEE Transactions on Image Processing, 20:382-390

6. Tian J, Ma K K (2011) A survey on super-resolution imaging, SIViP, 5:329-342.

7. Qin F Q, He X H, Chen W L, Yang X M, Wu W (2009) Video super-resolution reconstruction based on sub-pixel registration and iterative back projection. Journal of Electronic Imaging, 18(1):1-11

8. Zhou F, Yang W M, Liao Q M (2012) A coarse-to-fine subpixel registration method to recover local perspective deformation in the application of image super-resolution. IEEE Transatctions on Image Processing, 21(1):53-66.

9. Ignacio I, Mohamed E B, Jordi A, Daniel C, Xavier D, Maxime D (2012) PSF shaping using adaptive optics for three dimentsional single molecule super resolution imaging and tracking. Optics Express, 20(5):4957-4967. 